\title{
Assessment of Post-Stroke Fatigue: The Fatigue Scale for Motor and Cognitive Functions
}

\author{
Martina Hubacher ${ }^{a}$ Pasquale Calabrese $^{\mathrm{a}}$ Claudio Bassetti $^{\mathrm{b}}$ Antonio Carota $^{\mathrm{c}}$ \\ Markus Stöcklin ${ }^{a}$ Iris-Katharina Penner ${ }^{a}$ \\ ${ }^{a}$ Department of Cognitive Psychology and Methodology, University of Basel, Basel, ${ }^{b}$ Department of Neurology, \\ University Clinic Inselspital Bern, Bern, 'Neurocentre GSMN, Genolier, Switzerland
}

\section{Key Words}

Post-stroke fatigue $\cdot$ Stroke $\cdot$ Post-stroke depression • Cognition

\begin{abstract}
Background/Aims: Post-stroke fatigue (PSF) is an important but still controversial issue since knowledge on its nature is still humble. The aim of the present study was to characterize PSF beyond the subacute phase. Methods: Thirty-one stroke patients (gender: 6 female, 25 male; age range: 35-76 years; 28 patients with ischemic stroke, 3 patients with hemorrhagic stroke; mean delay after stroke: $50.65 \pm 31.57$ days) were recruited and assessed by measures of fatigue (Fatigue Scale for Motor and Cognitive Functions [FSMC], Fatigue Severity Scale, and Modified Fatigue Impact Scale), depression (Beck Depression Inventory Fast Screen), cognition (Brief Repeatable Battery of Neuropsychological Tests) and upper and lower extremity functions (Nine-Hole Peg Test and 25-foot walk). Results: Depending on the different scales, PSF prevalence ranged from 16.1 to $58.1 \%$. Depression measures correlated significantly $(r(29) \geq 0.46 ; p<0.01)$ with the results of all fatigue scales. Seventy-one percent of patients showed cognitive deficits in at least one cognitive domain. Cognitive
\end{abstract}

fatigue measured by one subscale of the FSMC correlated most significantly with mental speed, working memory, and verbal short-term memory, while the motor subscale was associated with upper and lower extremity functions, mental speed, visual short-term memory, and working memory. A differentiation between lesion localization and fatigue severity in the motor or cognitive domain was only possible when applying the FSMC. Patients with cortical lesions scored higher on the cognitive subscale, while patients with subcortical lesions showed higher physical subscale scores. Conclusion: The present pilot study revealed differences between lesion localization and subdomains of fatigue after stroke by applying a new fatigue scale (FSMC). The results underline the necessity for separate assessment of motor and cognitive fatigue in stroke patients.

Copyright $\odot 2012$ S. Karger AG, Basel

\section{Introduction}

Post-stroke fatigue (PSF) prevalence varies between 30 and $72 \%[1-3]$ and affects more women $[2,4,5]$. PSF seems to be a very long-lasting symptom. Over 1 year after stroke half of the patients still experience fatigue [6],

\section{KARGER \\ Fax +41613061234 E-Mail karger@karger.ch} www.karger.com

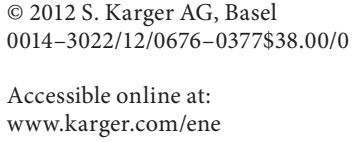

Dr. Iris-Katharina Penner

Department of Cognitive Psychology and Methodology

University of Basel

Missionsstrasse 60/62, CH-4055 Basel (Switzerland)

Tel. +41 61267 3525, E-Mail ik.penner@unibas.ch 
whereas other symptoms improve much faster during the rehabilitation process. In addition, $40-50 \%$ of stroke patients report that fatigue is their main complaint or one of their most debilitating symptoms [6, 7].

Unfortunately, today there is still relatively limited knowledge on the pathophysiology of PSF and the relation to comorbidities, such as depression and cognitive deficits [8]. Although PSF is of a subjective nature, for its mental as well as for its physical components, studies published so far focused only on the physical components, mostly neglecting the cognitive part. A more elaborated differentiation of the lesion sites might help to learn more about the pathological mechanisms responsible for the different clinical characteristics. While some studies could not find any relation between lesion site and PSF [7, 9], Staub and Bogousslavsky [10], based on a pilot study, proposed a cortico-subcortical gradient in frequency to develop fatigue from low frequency in cortical lesions to a higher frequency in patients with subcortical and brainstem lesions. This assumption was substantiated by a recent study from Tang et al. [11], who found more basal ganglia infarcts in patients with fatigue than in non-fatigued individuals.

Fatigue is often considered as a symptom of poststroke depression (PSD), an assumption that is supported by the evidence of significant correlations between scores of fatigue severity and depression [4, 12-15]. However, findings of other studies suggest that PSF and PSD might be independent entities since PSF can occur in stroke patients without any signs of depression $[7,15]$.

Taken together, little knowledge is still available on the nature of PSF and related aspects. This might not only be due to the unknown pathophysiological processes but also due to the psychometric properties of the applied fatigue instruments so far. Since fatigue is a purely subjective symptom and since objective measures to quantify and diagnose are missing, a reliable assessment depends on psychometric measures (fatigue questionnaires). The problem of many existing fatigue scales, however, is that they have methodological limitations which are related to scale construction and validation. Thus, the aim of the present pilot study was to characterize fatigue over the subacute phase of stroke by three different fatigue instruments, the Fatigue Severity Scale (FSS) [16], the Modified Fatigue Impact Scale (MFIS) [17], and the Fatigue Scale for Motor and Cognitive Functions (FSMC) [18], a new fatigue instrument dedicated to a differentiation between cognitive and motor fatigue, and to relate our fatigue findings to measures of depression and cognitive performance.

\section{Methods}

\section{Participants}

Between 2007 and 2008, 31 stroke patients (6 women and 25 men) with stroke were recruited from eight rehabilitation centers (Kantonsspital Bruderholz, RehaClinic Braunwald, Neurologische Klinik Elzach, Berner Klinik Montana, Reha-Klinik Walenstadtberg, Klinik Valens, Rheinburgklinik, and Kliniken Schmieder Allensbach) in Switzerland and Germany. Patients were between 35 and $76(59.29 \pm 10.30)$ years old. Only patients without any other neurological or psychiatric diseases, as revealed in medical records and actual anamnesis, were included. Additional exclusion criteria were clinical apparent aphasia and neglect to ensure full answers' validity to questionnaires. Twenty-eight patients suffered from ischemia and 3 from hemorrhage. Patient characteristics with respect to age, gender, educational level, stroke event, and stroke localization are shown in table 1 . All subjects gave written informed consent. The study was approved by the local ethics committee of the University of Basel.

Fatigue Assessment and Neuropsychological Examination

Neuropsychological examination was performed on average $50.65 \pm 31.57$ days after stroke by the Brief Repeatable Battery of Neuropsychological Tests (BRB-N) [19] composed of tests of verbal and visual short- and long-term memory, information processing speed, working memory, and executive functions. Upper and lower extremity functions were assessed by the Nine-Hole Peg test (9-HPT) and the 25-foot walk measure [20], respectively. For each patient, the whole assessment procedure took $2 \mathrm{~h}$. For statistical comparisons, $\mathrm{z}$ values for all tests were calculated [21, 22]. In contrast to Scherer et al. [21], BRB-N z values lower than -1.65 were rated as cognitively impaired since this cutoff represents the $90 \%$ confidence interval.

Fatigue was assessed by two well-known instruments, the FSS [16] and the MFIS [17], as well as by a recently developed scale, the FSMC [18]. The psychometric properties of the FSMC are excellent with Cronbach's $\alpha$, as a measure for internal consistency, of 0.95 for the total scale, 0.93 for the cognitive subscale, and 0.91 for the motor subscale. In a large validation study, the scale was evaluated on 309 multiple sclerosis patients and 147 healthy controls. In a direct comparison with the FSS and MFIS, a ROC analysis revealed that sensitivity and specificity were highest for the FSMC.

Twenty-four patients ( 4 women and 20 men) accepted to participate in a retest for fatigue assessment after 4 weeks. All patients were back at home at this time. This information was used to assess stability of PSF symptoms.

Depression was assessed by the Beck Depression Inventory (BDI) [23]. To avoid somatic interdependency between stroke, depression, and fatigue we excluded all somatic items in accordance to the BDI-Fast Screen for further analysis (BDI-FS [24]). Further, information on stroke event was based upon visual inspection of actual MRI scans of the individual patient. Stroke classification of affected territories comprised a cortical/subcortical subdivision. 
Table 1. Patient characteristics

\begin{tabular}{|c|c|c|}
\hline & Mean & SD \\
\hline \multirow[t]{2}{*}{ Age, years } & 59.29 & 10.30 \\
\hline & $\mathrm{n}$ & $\%$ \\
\hline \multicolumn{3}{|l|}{ Gender } \\
\hline Female & 6 & 19 \\
\hline Male & 25 & 81 \\
\hline \multicolumn{3}{|l|}{ Educational level } \\
\hline Secondary school & 5 & 16 \\
\hline College & 20 & 65 \\
\hline University & 6 & 19 \\
\hline \multicolumn{3}{|l|}{ Stroke event } \\
\hline First & 23 & 74 \\
\hline Second & 4 & 13 \\
\hline Third & 2 & 7 \\
\hline \multicolumn{3}{|l|}{ Stroke type } \\
\hline Ischemia & 28 & 90 \\
\hline Intracerebral hemorrhage & 3 & 10 \\
\hline \multicolumn{3}{|l|}{ Stroke localization } \\
\hline Cortical & 6 & 19 \\
\hline Left hemisphere & 1 & 3 \\
\hline Right hemisphere & 4 & 13 \\
\hline Both hemispheres & 1 & 3 \\
\hline Subcortical & 19 & 62 \\
\hline Left hemisphere & 7 & 23 \\
\hline Right hemisphere & 8 & 26 \\
\hline Both hemispheres & 2 & 6 \\
\hline Unknown & 2 & 6 \\
\hline Cortical and subcortical & 6 & 19 \\
\hline Left hemisphere & 3 & 10 \\
\hline Right hemisphere & 3 & 10 \\
\hline Both hemispheres & 0 & 0 \\
\hline
\end{tabular}

\section{Results}

\section{Assessment of Fatigue}

When applying the FSS, 5 patients (16.1\%) were above the critical cutoff of 4.6 for fatigue [25], whereas when using the MFIS, 11 patients (35.5\%) reached clinically relevant values. However, when applying the FSMC, $58.1 \%$ of patients turned out to have clinically relevant fatigue symptoms of different severity grades. Detailed information on fatigue prevalence and severity measured with the FSMC is given in table 2 .

In the 24 patients who participated in the retesting 4 weeks later, fatigue scores measured with the FSS and the MFIS remained stable. In contrast, when fatigue was measured with the FSMC, fatigue symptoms obviously worsened over time. Patients showed significantly in-
Table 2. Fatigue prevalence and severity measured with the FSMC

\begin{tabular}{|c|c|c|c|c|c|c|}
\hline & \multicolumn{2}{|c|}{$\begin{array}{l}\text { FSMC-C } \\
(\mathrm{n}=31)\end{array}$} & \multicolumn{2}{|c|}{$\begin{array}{l}\text { FSMC-M } \\
(\mathrm{n}=31)\end{array}$} & \multicolumn{2}{|c|}{$\begin{array}{l}\text { FSMC-S } \\
(\mathrm{n}=31)\end{array}$} \\
\hline & $\mathrm{n}$ & $\%$ & $\mathrm{n}$ & $\%$ & $\mathrm{n}$ & $\%$ \\
\hline No fatigue & 15 & 48.4 & 12 & 38.7 & 13 & 41.9 \\
\hline Mild fatigue & 7 & 22.6 & 6 & 19.4 & 6 & 19.4 \\
\hline Moderate fatigue & 5 & 16.1 & 5 & 16.1 & 7 & 22.6 \\
\hline Severe fatigue & 4 & 12.9 & 8 & 25.8 & 5 & 16.1 \\
\hline
\end{tabular}

FSMC-C = Cognitive subscale; FSMC-M = motor subscale; FSMC-S = sum score of scale.

Table 3. Fatigue scores measured at baseline and after 4 weeks

\begin{tabular}{|c|c|c|c|c|c|c|}
\hline & \multicolumn{2}{|c|}{ Baseline } & \multicolumn{2}{|c|}{ After 4 weeks } & \multirow[b]{2}{*}{$\mathrm{t}$} & \multirow[b]{2}{*}{$\mathrm{p}$} \\
\hline & Mean & SD & Mean & SD & & \\
\hline FSS & 3.00 & 0.32 & 3.73 & 0.39 & -1.81 & 0.083 \\
\hline MFIS & 26.79 & 3.86 & 32.21 & 4.27 & -1.18 & 0.252 \\
\hline FSMC-C & 19.63 & 1.61 & 24.92 & 2.12 & -2.40 & 0.025 \\
\hline FSMC-M & 22.67 & 1.74 & 28.00 & 2.21 & -2.22 & 0.037 \\
\hline FSMC-S & 42.29 & 3.06 & 52.92 & 4.18 & -2.34 & 0.028 \\
\hline
\end{tabular}

FSMC-C = Cognitive subscale; FSMC-M = motor subscale; FSMC-S = sum score of scale.

creased scores after 4 weeks on both subscales as well as an increased FSMC total score (see table 3 for details).

\section{Relation between Depression and Fatigue}

Mean depression score on BDI-FS was $1.94 \pm 2.32$. Twenty-five patients (81\%) showed no signs of depressive symptoms, 5 patients (16\%) were classified with mild depressive symptoms, and only 1 patient (3\%) scored in the range of severe depression. The BDI-FS score correlated significantly with the FSS score $(r(29)=0.46, \mathrm{p}=0.009)$, the MFIS score $(r(29)=0.62, \mathrm{p}<0.001)$, as well as all subscores of the FSMC (cognitive: $r(29)=0.61, \mathrm{p}<0.001$; motor: $r(29)=0.54, \mathrm{p}=0.002$; sum score: $r(29)=0.61, \mathrm{p}<$ $0.001)$.

\section{Stroke Localization and Fatigue}

Six patients had cortical lesions, 19 suffered from subcortical lesions, and 6 patients had mixed subcortical and cortical lesions. In figure 1 , mean values of the fatigue subscales for the three lesion localization groups are diagrammed. In the cognitive subscale of the FSMC, patients 


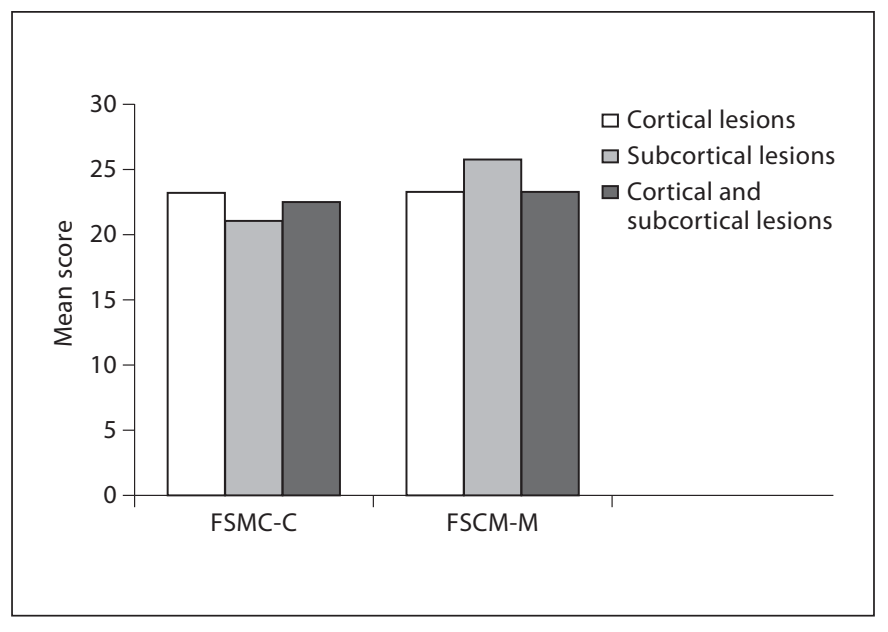

Fig. 1. Mean scores for FSMC-C (subscale for cognitive fatigue) and FSMC-M (subscale for physical fatigue) for all three types of lesions.

with cortical lesions reached the highest values, while patients with subcortically located lesions reached the lowest. In the physical subscale of the FSMC this effect changed: patients with cortical lesions showed less fatigue symptoms than patients with subcortical lesions. Patients with mixed lesions showed values between the other two groups. The FSMC total score did not differ between the three groups (not diagrammed). This association between lesion localization and differentiated fatigue symptoms could not be detected by using the subscales of MFIS. Here, patients with subcortically located lesions always reached the highest fatigue values (see table 4 for further information).

In table 4, means and SD of the FSMC and MFIS scores for the three lesion localization subgroups are displayed. Due to the small number of patients, Cohen's $d$ [26] for effect sizes of group differences between patients with cortical and subcortical lesions was calculated. For this purpose, the mean differences of scores between patients with cortically and patients with subcortically located lesions were divided by the pooled SD

$$
d=\frac{M_{\text {cortical }}-M_{\text {subcortical }}}{\sqrt{\frac{S D_{\text {cortical }}{ }^{2} \cdot\left(n_{\text {cortical }}-1\right)+S D_{\text {subcortical }}{ }^{2} \cdot\left(n_{\text {subcortical }}-1\right)}{n_{\text {cortical }}+n_{\text {subcortical }}-2}}} .
$$

To evaluate the effect sizes, $d=0.20$ was rated as a small effect, $d=0.50$ as a moderate effect, and $d=0.80$ as a large effect. By applying this statistical procedure, small effect sizes were found for the differences in FSMC scores between cortically and subcortically located lesions on both subdomains of the FSMC, whereas the sum score did not differ in both of these groups of patients (see table 4 for more details).

\section{Relation between Cognition, Upper and Lower Extremity Functions and Fatigue}

Twenty-two patients (71\%) showed reduced performance in one or more cognitive subtests of the BRB-N. Most deficits were observed in executive functions followed by mental speed, verbal memory, and working memory. Sixty-one percent of patients showed clinically relevant upper extremity, and $10 \%$ lower extremity dysfunctions. Table 5 shows means and SD for the cognitive performance and the percentage of patients having clinically meaningful deficits on each cognitive domain and upper and lower extremity functions.

To assess relations between fatigue and cognitive functioning, bivariate correlations were calculated. As shown in table 6, FSS scores neither correlated significantly with any cognitive measures nor with those of upper and lower extremity functions. The cognitive subscale of the MFIS correlated with verbal short-term memory, mental speed, and executive functions, while the physical subscale was only related to mental speed but not to motor functions. The cognitive subscale of the FSMC was related to verbal short-term memory, mental speed, and working memory, while the motor subscale was related to cognitive domains, such as visual short-term memory, mental speed, and working memory, but also to upper and lower extremity functions, measured with the 9-HPT and 25 -foot walk (see table 6 for details).

\section{Sensitivity and Specificity of the Different Fatigue \\ Scales}

A logistic regression analysis was computed for the three applied fatigue instruments to evaluate sensitivity and specificity of the scales. For this purpose, 31 agematched healthy controls from our large database on the FSMC and the 31 stroke patients were included in the logistic regression. Hereby, the percentage of patients correctly diagnosed as stroke patients ('sensitivity') and the percentage of controls correctly classified as not having stroke ('specificity') could be determined. Thus, specificity and sensitivity were analyzed with respect to 'stroke diagnosis'. Since PSF is a well-known symptom in stroke patients that by definition should not be present in healthy subjects, the terms 'sensitivity and specificity' refer to the ability of the different scales to relate fatigue to the underlying diagnosis of stroke. The FSS reached the lowest values (sensitivity: $51.6 \%$; specificity: $61.3 \%$ ), while the 
Table 4. Mean FSMC and MFIS scores for the three lesion localization groups

\begin{tabular}{|c|c|c|c|c|c|c|c|}
\hline & \multicolumn{2}{|c|}{$\begin{array}{l}\text { Cortical and sub- } \\
\text { cortical localization }\end{array}$} & \multicolumn{2}{|c|}{$\begin{array}{l}\text { Cortical } \\
\text { localization }\end{array}$} & \multicolumn{2}{|c|}{$\begin{array}{l}\text { Subcortical } \\
\text { localization }\end{array}$} & \multirow[t]{2}{*}{$d$} \\
\hline & Mean & SD & Mean & SD & Mean & SD & \\
\hline FSMC-C & 22.50 & 4.81 & 23.17 & 11.07 & 21.05 & 9.81 & 0.21 \\
\hline FSMC-M & 23.33 & 4.97 & 23.33 & 11.62 & 25.74 & 9.92 & -0.23 \\
\hline FSMC-S & 45.83 & 9.11 & 46.50 & 22.56 & 46.79 & 18.42 & -0.01 \\
\hline MFIS-C & 14.83 & 9.06 & 9.33 & 10.25 & 16.37 & 9.26 & -0.74 \\
\hline MFIS-M & 14.50 & 10.29 & 9.83 & 9.45 & 11.68 & 9.89 & -0.19 \\
\hline MFIS-S & 32.33 & 19.67 & 21.00 & 21.28 & 31.63 & 19.46 & -0.53 \\
\hline
\end{tabular}

FSMC-C = Cognitive subscale; FSMC-M = motor subscale; FSMC-S = sum score of scale; MFIS-C = cognitive subscale; MFIS-M = motor subscale; MFIS-S = sum score.

MFIS (sensitivity: $67.7 \%$; specificity: $71.0 \%$ ) and the FSMC (sensitivity: 64.5\%; specificity: $71.0 \%$ ) reached comparable values.

\section{Discussion}

\section{Fatigue Prevalence and Depression}

A comparison between FSMC and two other wellestablished fatigue scales (FSS and MFIS) revealed that fatigue prevalence rates were highest $(58.1 \%)$ when the FSMC was applied. Obviously, the graduation system of the FSMC with cutoff values for mild, moderate, and severe fatigue offers a more accurate evaluation of the mild fatigue symptoms than the overall cutoff of the MFIS.

Test-retest results of the FSMC revealed an increase in fatigue severity. PSF worsening within 4 weeks can be explained by cognitive processes of realizing that one is not yet back to normal and that recovery takes much longer than expected. In a study by Christensen et al. [27], slight improvement of fatigue symptoms was described after 3 months, supporting the idea that reduction of PSF symptoms needs time.

In the present study, significant correlations between PSF and PSD were found. However, the majority of patients did not show clinically relevant PSD, although $>58 \%$ were diagnosed as having PSF. In contrast to previous studies, in our study, we applied the BDI-FS where the physical items related to depression are excluded. Thus, the lower amount of patients being diagnosed with PSD can in part be due to this issue. Of much more interest is the finding that, obviously, PSF can occur in patients
Table 5. Cognitive performance and upper and lower extremity functions

\begin{tabular}{|c|c|c|c|}
\hline & Mean & $\mathrm{SD}$ & $\begin{array}{l}\text { Clinically } \\
\text { relevant } \\
\text { values, \% }\end{array}$ \\
\hline \multicolumn{4}{|l|}{ Verbal short-term memory } \\
\hline SRT-LTS & -0.41 & 1.52 & 23 \\
\hline SRT-CLTR & -0.36 & 1.27 & 16 \\
\hline \multicolumn{4}{|l|}{ Verbal long-term memory } \\
\hline SRT-DR & -1.10 & 1.80 & 39 \\
\hline \multicolumn{4}{|l|}{ Visual short-term memory } \\
\hline 10/36 Spatial Recall Test & 0.24 & 1.15 & 3 \\
\hline \multicolumn{4}{|l|}{ Visual long-term memory } \\
\hline $10 / 36-\mathrm{DR}^{\mathrm{d}}$ & 0.16 & 1.30 & 10 \\
\hline \multicolumn{4}{|l|}{ Mental speed } \\
\hline SDMT & -1.14 & 1.46 & 36 \\
\hline \multicolumn{4}{|l|}{ Executive functions } \\
\hline Word list generation & -1.39 & 0.87 & 42 \\
\hline \multicolumn{4}{|l|}{ Working memory } \\
\hline PASAT & -1.11 & 1.33 & 26 \\
\hline \multicolumn{4}{|l|}{ Upper extremity } \\
\hline 9-HPT & -1.30 & 1.09 & 61 \\
\hline \multicolumn{4}{|l|}{ Lower extremity } \\
\hline 25-foot walk ${ }^{\mathrm{a}}$ & 0.22 & 0.31 & 10 \\
\hline
\end{tabular}

SRT-LTS = Selective Reminding Test - Long-Term Storage; SRT-CLTR $=$ Selective Reminding Test - Consistent Long-Term Retrieval; SRT-DR = Selective Reminding Test - Delayed Recall; 10/36-DR $=$ Spatial Recall Test - Delayed Recall; SDMT $=$ Symbol Digits Modalities Test; PASAT $=$ Paced Auditory Serial Addition Test $(\mathrm{n}=26) .{ }^{\mathrm{a}} \mathrm{n}=28$. 
Table 6. Correlations between fatigue and cognitive function, and upper and lower extremity functions

\begin{tabular}{|c|c|c|c|c|c|c|c|}
\hline & FSS & MFIS-C & MFIS-M & MFIS-S & FSMC-C & FSMC-M & FSMC-S \\
\hline \multicolumn{8}{|l|}{ Verbal short-term memory } \\
\hline SRT-LTS & -0.18 & $-0.38 *$ & -0.29 & $-0.36^{*}$ & $-0.39^{*}$ & -0.36 & -0.35 \\
\hline SRT-CLTR & -0.16 & $-0.38 *$ & -0.34 & $-0.39^{*}$ & $-0.36^{*}$ & -0.22 & -0.31 \\
\hline \multicolumn{8}{|l|}{ Verbal long-term memory } \\
\hline SRT-DR & -0.28 & -0.33 & -0.22 & -0.30 & -0.34 & -0.17 & -0.27 \\
\hline \multicolumn{8}{|l|}{ Visual short-term memory } \\
\hline 10/36 Spatial Recall Test & -0.08 & -0.05 & -0.22 & -0.14 & -0.26 & $-0.39^{*}$ & -0.34 \\
\hline \multicolumn{8}{|l|}{ Visual long-term memory } \\
\hline $10 / 36-\mathrm{DR}$ & -0.18 & -0.08 & -0.22 & -0.14 & -0.26 & -0.34 & -0.32 \\
\hline \multicolumn{8}{|l|}{ Mental speed } \\
\hline SDMT & -0.23 & $-0.45^{*}$ & $-0.39^{*}$ & $-0.44^{*}$ & $-0.54^{* *}$ & $-0.43^{*}$ & $-0.52^{* *}$ \\
\hline \multicolumn{8}{|l|}{ Executive functions } \\
\hline Word list generation & -0.14 & $-0.41^{*}$ & -0.31 & $-0.41^{*}$ & -0.33 & -0.05 & -0.20 \\
\hline \multicolumn{8}{|l|}{ Working memory } \\
\hline PASAT & -0.21 & -0.25 & -0.08 & -0.17 & $-0.49^{*}$ & $-0.44^{*}$ & $-0.50^{* *}$ \\
\hline \multicolumn{8}{|l|}{ Upper extremity } \\
\hline 9-HPT & 0.02 & 0.13 & 0.04 & 0.23 & -0.12 & $-0.40^{*}$ & -0.28 \\
\hline \multicolumn{8}{|l|}{ Lower extremity } \\
\hline 25-foot walk ${ }^{\mathrm{a}}$ & -0.37 & -0.14 & -0.18 & -0.15 & -0.35 & $-0.43^{*}$ & $-0.41^{*}$ \\
\hline
\end{tabular}

MFIS-C = Cognitive subscale; MFIS-M = motor subscale; $\mathrm{MFIS}-\mathrm{S}=$ sum score of scale; FSMC-C = cognitive subscale; FSMC-M = motor subscale; FSMC-S = sum score of scale; SRT-LTS = Selective Reminding Test - Long-Term Storage; SRT-CLTR = Selective Reminding Test - Consistent Long-Term Retrieval; SRT-DR = Selective Reminding Test - Delayed Recall; 10/36-DR = Spatial Recall Test - Delayed Recall; SDMT $=$ Symbol Digits Modalities Test; PASAT $=$ Paced Auditory Serial Addition Test $(\mathrm{n}=26) .{ }^{\mathrm{a}} \mathrm{n}=28$.

${ }^{*} \mathrm{p}<0.05 ;{ }^{* *} \mathrm{p}<0.01$.

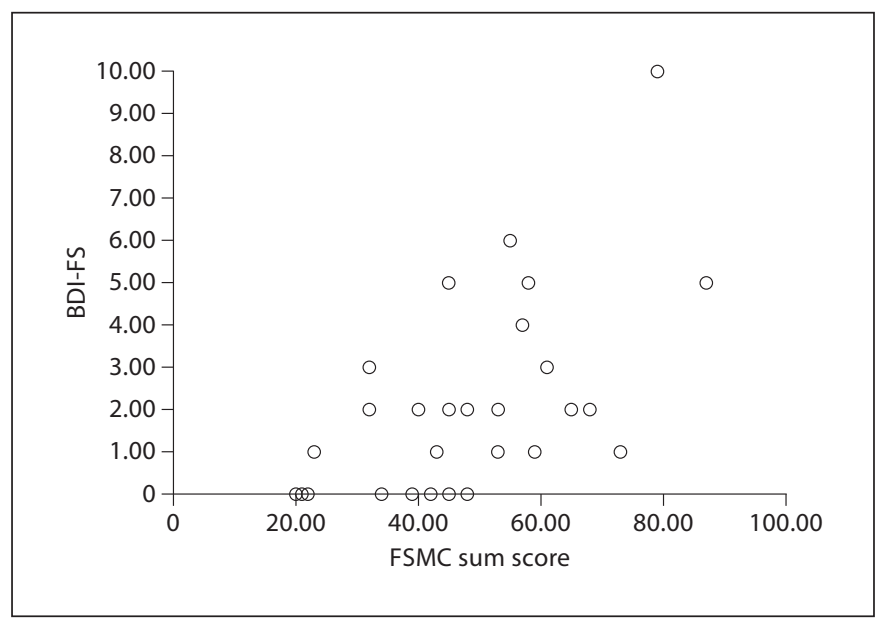

Fig. 2. Values of each patient for depression (BDI-FS) and fatigue (FSMC sum score). While there are no patients showing signs of depression independently of fatigue, the opposite relation was detected: patients with fatigue, in most of the cases, also show signs of depression. without showing any signs of depression. Further, figure 2 illustrates that the opposite relation seems to be less likely, leading to the hypothesis that PSD might be regarded as a feature of PSF. Thus, in summary, these results clearly demonstrate the necessity to assess the two symptoms reliably to provide the right diagnosis and therapeutic approach to the patients.

\section{Lesion Localization and Fatigue}

Regarding the question to which extent the localization of the lesion influences the occurrence of fatigue, we found that patients with subcortical lesions showed higher MFIS scores on all subscales compared to patients with cortical lesions. This result is in accordance with the theory proposed by Staub and Bogousslavsky [10]. Contrarily, the results of the FSMC revealed another more differentiated picture that was not detectable by the MFIS: patients with subcortical lesions showed higher scores on motor fatigue, while cognitive fatigue was more frequent in patients with cortical lesions. The small but relevant effect sizes for the differences in fatigue scores between subcortical and cortical lesions elucidate the necessity of 
a differentiation between cognitive and motor fatigue to better understand the pathophysiology of PSF. This result is of importance insofar as it underlines for the first time in a sample of stroke patients that cognitive and motor fatigue are probably different pathophysiological entities where cognitive fatigue can be assumed to be of cortical and motor fatigue of subcortical origin.

Other studies, however, could not find any significant relation between stroke localization and fatigue $[5,14$, 28]. This might primarily be due to the applied fatigue instruments which either did not differentiate between motor and cognitive fatigue or were less sensitive than the applied FSMC. These differences, due to the sensitivity of the fatigue scales, are also reflected in our study when comparing FSMC to MFIS results.

\section{Cognition, Upper and Lower Extremity Functions, and Fatigue}

Seventy-one percent of subjects showed cognitive impairment mainly in the domains of executive functions, mental speed, and memory. Using FSS and MFIS for assessing fatigue, none or only a few correlations, respectively, were found between fatigue level and cognitive performance. However, when the FSMC was applied, cognitive fatigue turned out to be significantly related to the performance in several cognitive domains, whereas physical fatigue was mainly related to upper and lower extremity functions but additionally also to cognitive measures assessing speed and stamina. It may be intuitively right that cognitive fatigue is mainly reflected by neuropsychological outcomes and motor fatigue mainly by physical functioning. However, it has to be noted that these correlations are barely seen in patients with multiple sclerosis [16]. In the present study, cognitive fatigue seems to be mainly associated with cognitive functioning, while motor fatigue is not exclusively related to physical functioning but also to cognitive aspects, such as speed and stamina. As already reported for lesion localization, the association between motor and cognitive functions and cognitive and motor fatigue was only found when using the FSMC. The cognitive and physical subscales of the MFIS were not able to show the above-mentioned discrimination, although sensitivity and specificity values were comparable to the FSMC.

\section{Study Limitations}

Our study has several limitations. First, the sample size was rather small, and subgroup statistics on the interesting questions about stroke localization and occurrence and subtype of fatigue could only be performed by

non-parametric analyses and differences could only be quantified by effect sizes. Since differences were still detectable, these results encourage the performance of future studies on larger samples. Second, the numbers of patients in the subgroups with respect to lesion localization were not of equal size, thus making statistical comparisons difficult. Third, a more detailed classification of lesion localization would be warranted to better understand the origin of PSF. Fourth, 3 patients did not suffer from ischemia but had intracerebral hemorrhage. This might have an influence on cognitive performance, time of recovery after stroke event, and fatigue. Our sample, however, was too small to run meaningful subgroup statistics with respect to stroke type. Nevertheless, leaving out the 3 hemorrhagic patients would not have changed the pattern of results entirely. Fifth, more detailed lesion characteristics and more elaborated clinical data were not available, since the focus of our study was more on the methodological issues related to the new scale than on the characterization of the clinical population. This will be a further issue in future studies.

Despite these limitations, the present pilot study for the first time revealed differences between lesion localization and subdomains of fatigue after stroke by applying a new fatigue scale. So far, the results might be conclusive enough to change behavior in clinical practice towards separate assessment of motor and cognitive fatigue in patients after stroke.

\section{Acknowledgements}

We thank the following rehabilitation centers for participating in our study: Kantonsspital Bruderholz, RehaClinic Braunwald, Neurologische Klinik Elzach, Berner Klinik Montana, RehaKlinik Walenstadtberg, Klinik Valens, Rheinburgklinik, and Kliniken Schmieder Allensbach.

References

Eur Neurol 2012;67:377-384
1 de Groot MH, Philips SJ, Eskes GA: Fatigue associated with stroke and other neurologic conditions: implications for stroke rehabilitation. Arch Phys Med Rehabil 2003;84: 1714-1720.

2 Falconer M, Walsh S, Harbison JA: Estimated prevalence of fatigue following stroke and transient ischemic attack is dependent on terminology used and patient gender. J Stroke Cerebrovasc Dis 2010;19:431-434.

-3 Colle F, Bonan I, Gellez Leman M, Bradai N, Yelnik A: Fatigue after stroke. Ann Readapt Med Phys 2006;49:361-364. 
-4 Glader EL, Stegmayr B, Asplund K: Poststroke fatigue: a 2-year follow-up study of stroke patients in Sweden. Stroke 2002;33: 1327-1333.

5 Lynch J, Mead G, Greig C, Young A, Lewis S, Sharpe M: Fatigue after stroke: the development and evaluation of a case definition. J Psychosom Res 2007;63:539-544.

-6 van der Werf SP, van den Broek HLP, Anten HWM, Bleijenberg G: Experience of severe fatigue long after stroke and its relation to depressive symptoms and disease characteristics. Eur Neurol 2000;45:28-33.

7 Ingles JL, Eskes GA, Phillips SJ: Fatigue after stroke. Arch Phys Med Rehabil 1999;80:173178.

$>8$ Lerdal A, Bakken LN, Kouwenhoven SE, Pedersen G, Kirkevold M, Finset A, Kim HS: Poststroke fatigue - a review. J Pain Symptom Manage 2009;38:928-949.

$>9$ Leegaard OF: Diffuse cerebral symptoms in convalescents from cerebral infarction and myocardial infarction. Acta Neurol Scand 1983;67:348-355.

10 Staub F, Bogousslavsky J: Fatigue after stroke: a major but neglected issue. Cerebrovasc Dis 2001;12:75-81.

11 Tang WK, Chen YK, Mok V, Chu WC, Ungvari GS, Ahuja AT, Wong KS: Acute basal ganglia infarcts in poststroke fatigue: an MRI study. J Neurol 2010;257:178-182.

-12 Schepers VP, Visser-Meily AM, Ketelaar M, Lindeman E: Poststroke fatigue: course and its relation to personal and stroke-related factors. Arch Phys Med Rehabil 2006;87: 184-188.
13 Naess H, Nyland HI, Thomassen L, Aarseth J, Myhr KM: Fatigue at long-term follow-up in young adults with cerebral infarction. Cerebrovasc Dis 2005;20:245-250.

14 Appelros P: Prevalence and predictors of pain and fatigue after stroke: a populationbased study. Int J Rehabil Res 2006;29:329333.

15 Choi-Kwon S, Han SW, Kwon SU, Kim JS: Poststroke fatigue: characteristics and related factors. Cerebrovasc Dis 2005;19:84-90.

-16 Krupp LB, LaRocca NG, Muir-Nash J, Steinberg AD: The fatigue severity scale. Application to patients with multiple sclerosis and systemic lupus erythematosus. Arch Neurol 1989;46:1121-1123.

17 Multiple Sclerosis Council for Clinical Practice Guidelines: Fatigue and Multiple Sclerosis: Evidence-Based Management Strategies for Fatigue in Multiple Sclerosis. Washington DC, Paralyzed Veterans of America, 1998.

18 Penner IK, Raselli C, Stocklin M, Opwis K, Kappos L, Calabrese P: The Fatigue Scale for Motor and Cognitive Functions (FSMC): validation of a new instrument to assess multiple sclerosis-related fatigue. Mult Scler 2009;15:1509-1517.

19 Rao SM: A Manual for the Brief, Repeatable Battery of Neuropsychological Tests in Multiple Sclerosis. New York, National Multiple Sclerosis Society, 1990.

20 Cutter GR, Baier ML, Rudick RA, Cookfair DL, Fischer JS, Petkau J, Syndulko K, Weinshenker BG, Antel JP, Confavreux C, Ellison GW, Lublin F, Miller AE, Rao SM, Reingold S, Thompson A, Willoughby E: Development of a multiple sclerosis functional composite as a clinical trial outcome measure. Brain 1999; 122:871-882.
21 Scherer P, Baum K, Bauer H, Gohler H, Miltenburger C: Normierung der Brief Repeatable Battery of Neuropsychological Tests (BRB-N) für den deutschsprachigen Raum. Nervenarzt 2004;75:984-990.

22 Fischer JS, Rudick RA, Cutter GR, Reingold SC: The Multiple Sclerosis Functional Composite Measure (MSFC): an integrated approach to MS clinical outcome assessment. National MS Society Clinical Outcomes Assessment Task Force. Mult Scler 1999;5:244250.

23 Beck AT, Ward CH, Mendelson M, Mock J, Erbaugh J: An inventory for measuring depression. Arch Gen Psychiatry 1961;4:561571.

24 Beck A, Steer R, Brown G: BDI-Fast Screen for Medical Patients. The Psychological Corporation, San Antonio, TX, 2000.

25 Flachenecker P, Kumpfel T, Kallmann B, Gottschalk M, Grauer O, Rieckmann P, Trenkwalder C, Toyka K: Fatigue in multiple sclerosis: a comparison of different rating scales and correlation to clinical parameters. Mult Scler 2002;8:523-526.

26 Cohen J: Statistical Power Analysis for the Behavioral Sciences. Hillsdale, Lawrence Erlbaum Associates, 1988.

27 Christensen D, Johnsen SP, Watt T, Harder I, Kirkevold M, Andersen G: Dimensions of post-stroke fatigue: a two-year follow-up study. Cerebrovasc Dis 2008;26:134-141.

28 Carlsson GE, Moller A, Blomstrand C: Consequences of mild stroke in persons $<75$ years - a 1-year follow-up. Cerebrovasc Dis $2003 ; 16: 383-388$ 